\title{
ACTUALIZACION DE LA EVALUACION RADIOLOGICA DE LA ESCOLIOSIS
}

\author{
Drs. Jorge Díaz $J^{(1)}$, Carolina Schröter $\mathbf{G}^{(1)}$, Ronald Schulz $\mathbf{I}^{(2)}$. \\ 1. Centro de Imagenología, Hospital Clínico de la Universidad de Chile. Santiago, Chile. \\ 2. Servicio de Traumatología, Hospital Clínico de la Universidad de Chile. Santiago, Chile.
}

\section{UPDATE ON RADIOLOGIC ASSESSMENT OF SCOLIOSIS}

\begin{abstract}
Spinal deformities affect a considerable number of individuals of all ages. The etiological spectrum is broad and in cases of severe deformities a surgical treatment may be required.

In addition to clinical evaluation, full spine radiographs constitute a fundamental tool, both in the diagnostic process as well as in the therapeutic follow-up of these patients.

The aim of this paper is to provide a comprehensive overview on radiological assessment of spinal deformities, with an emphasis on scoliosis, by summarizing classical concepts and incorporating new ideas being developed in recent years, mainly due to the improvement and increased complexity of surgical management.
\end{abstract}

Keywords: Kyphosis, Scoliosis, Spinal curvatures.

Resumen: Las deformidades de columna vertebral afectan a un considerable número de individuos de todas las edades. Su espectro etiológico es amplio $y$ en casos de deformidades severas, el manejo de éstas puede llegar a ser quirúrgico.

Además de la evaluación clínica, la radiografía de columna total es un pilar fundamental, tanto en el proceso diagnóstico así como también en el control terapéutico de estos pacientes.

El objetivo de este artículo es dar una visión integral de la evaluación radiológica de las deformidades de columna, con énfasis en las escoliosis, recapitulando conceptos clásicos como también incorporando nociones nuevas que se han desarrollado en los últimos años, debido principalmente al progreso y aumento

Díaz J. y cols. Actualización de la evaluación radiológica de la escoliosis. Rev Chil Radiol 2009; 15: 141-151.

Correspondencia: jdiazjara@gmail.com

Trabajo recibido el 08 de marzo de 2009, aceptado para publicación el 12 de agosto 2009. de la complejidad de su manejo quirúrgico.

Palabras clave: Cifosis, Curvaturas de columna vertebral, Escoliosis.

\section{Introducción}

La columna vertebral (CV) es una compleja estructura osteo-fibro-cartilaginosa en la que, desde el punto de vista mecánico, los cuerpos vertebrales se disponen adoptando un eje relativamente recto en el plano coronal y ejes suavemente curvos en el plano sagital (cifosis y lordosis).

Tanto en el plano sagital como coronal, los ejes pueden sufrir deformidades respecto a los rangos descritos de normalidad, destacando entre estas alteraciones la escoliosis y el dorso curvo.

La evaluación clínica es el punto de partida para el diagnóstico de las deformidades de la CV; sin embargo siempre debe ser apoyada por una evaluación radiológica, que constituye uno de los pilares en el diagnóstico y control de evolución.

La mejor comprensión de la fisiopatología y el aumento en la complejidad del tratamiento quirúrgico de las escoliosis han dado origen a nuevas clasificaciones y formas de descripción, que se apoyan en la evaluación por radiología simple. Es así como ésta ha ido incorporando nuevos conceptos y remodelando los ya existentes, en mayor o en menor medida.

El objetivo del presente artículo es dar una visión actualizada respecto de la evaluación de la "radiografía de columna total", usando como referencia los conceptos más recientes del manejo clínico-quirúrgico de las escoliosis, que debieran ser el marco para el análisis de dicho examen.

\section{Definición}

La escoliosis es una desviación de la CV con características tridimensionales, cuya deformidad predominante es en el plano coronal (derechaizquierda). Sin embargo, tiene componentes rotacionales asociados así como también deformidades en el plano sagital, que pueden afectar la cifosis dorsal y la lordosis lumbar ${ }^{(1,2,4)}$. 
El examen clínico (test de Adams, asimetría del triángulo de la talla, desnivel de hombros, etc) es la base para la pesquisa de la escoliosis. Si se observa una asimetría paravertebral al examinar al paciente con el tronco flectado, la indicación sería una evaluación con radiología simple para confirmar y cuantificar la magnitud de la escoliosis. La radiografía también es la herramienta para el control de evolución, por lo que ésta debe ser adecuadamente adquirida y evaluada ${ }^{(4,10)}$.

\section{Radiografía de columna total Aspectos técnicos}

Los protocolos deben seguir ciertas normas, para así obtener estudios reproducibles y comparables que proporcionen toda la información necesaria para el diagnóstico:

1. La radiografía de columna completa se adquiere con un tubo de rayos $X$ estándard, a una distancia de alrededor 1,80-2 m (telerradiografía), con el haz centrado en el área T6-T7 ${ }^{(13)}$.

2. Para la proyección frontal (AP), el paciente se ubica de pies, con las rodillas extendidas y los pies separados a la distancia de los hombros, mirando recto hacia el frente. Es necesario que en la proyección frontal aparezca la totalidad de las crestas ilíacas, especialmente en los pacientes adolescentes, ya que éstas son un índice de maduración ósea (índice de Risser), que es parte importante en la decisión del manejo médico o quirúrgico ${ }^{(2)}$ (Figura 1). En caso de que

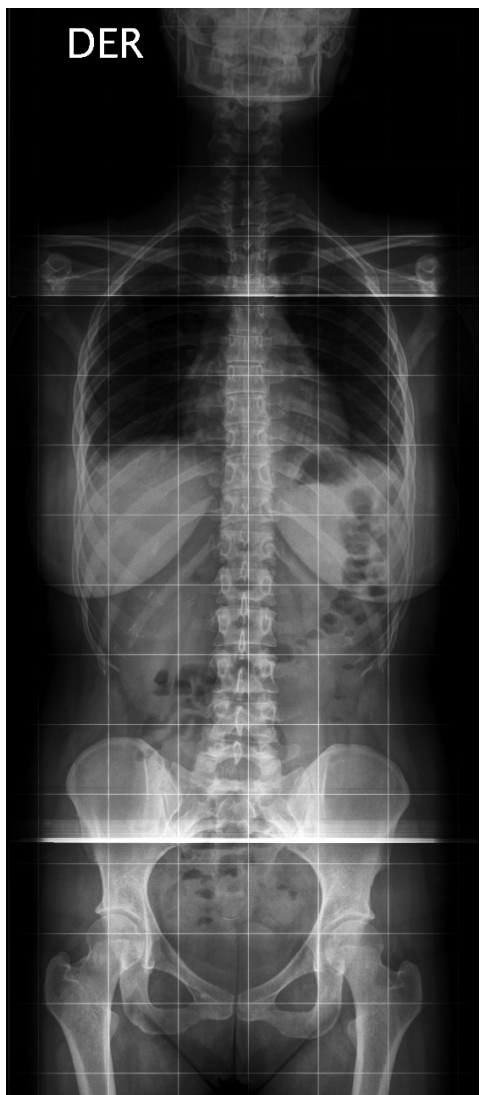

Figura 1. Radiografía de columna total $A P$. El examen cubre toda la columna vertebral, siendo además visibles las crestas ilíacas y las articulaciones coxofemorales. En este paciente no existe escoliosis. exista una discrepancia en la longitud de las extremidades inferiores mayor a $2 \mathrm{~cm}$ (medida a nivel de cabezas femorales), una evaluación más objetiva de las curvas se obtendría con una proyección frontal adicional con elevación del talón para nivelar la pelvis ${ }^{(3)}$.

3. En la proyección lateral, los codos permanecen flectados y los dedos encajados en las fosas supraclaviculares a ambos lados, o con los antebrazos descansando sobre un soporte anterior con lo cual se logra adecuada posición del tórax y desproyección de los brazos. Se recomienda que la elevación de los brazos no sea mayor a $30^{\circ}$ hacia anterior, respecto de la vertical(11) (Figura 2).

4. Existen proyecciones adicionales, entre ellas las radiografías con inclinación lateral (sidebending), necesarias para evaluar la flexibilidad de las curvas y su grado de corrección. El análisis de estas radiografías consiste en cuantificar el grado de reducción de las escoliosis cuando el paciente está inclinado hacia el lado convexo de las curvas, catalogándolas en estructurales y no estructurales de acuerdo a esto (Figura 3 ). Se adquieren en posición supina, con la mayor inclinación posible del paciente hacia uno y otro lado $^{(2,3)}$. Cabe destacar que la inclinación debe ser máxima, por lo que idealmente, debiera hacerse con la asistencia de dos personas: una que fije la pelvis, y otra que haga la presión en la dirección del bending, a la vez que ejerce una contrapresión en la zona apical de la curva. Es

F i g u r 2. Radiografía de columna total lateral. El examen cubre toda la columna vertebral y la elevación de los brazos es menor de $30^{\circ}$ respecto a la vertical. La cifosis torácica y lordosis lumbar se encuentran en rangos normales.

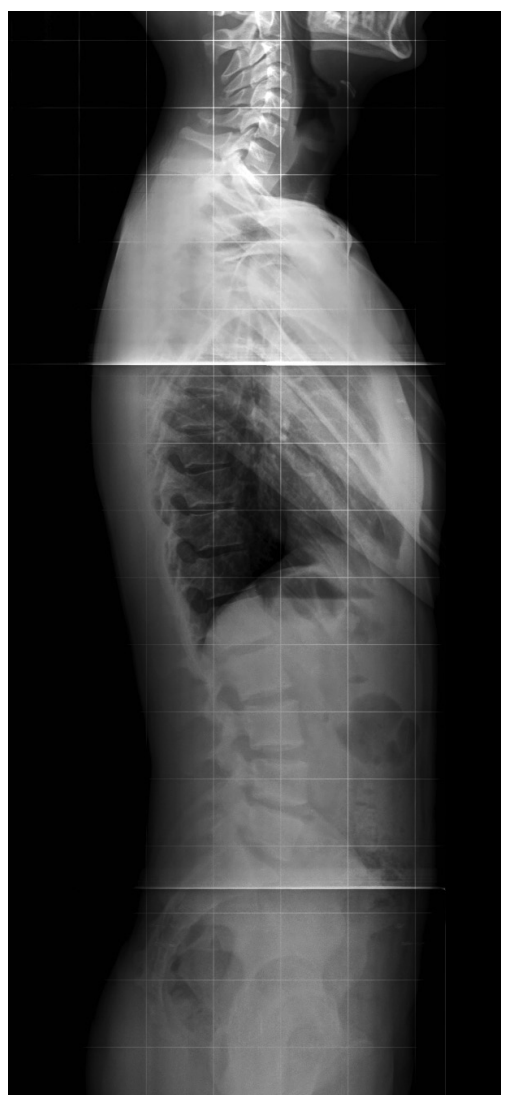


usual que el paciente salga del área de proyección del chasis; para solucionar este inconveniente, es recomendable tomar proyecciones bending de cada curva en forma individual. Debe tenerse en cuenta la importancia de que las proyecciones con inclinación lateral sean bien realizadas, ya que radiografías con inclinación submáxima pueden llevar a error en la apreciación del grado de rigidez de la curva y determinar errores en las estrategias quirúrgicas, como por ejemplo una liberación quirúrgica anterior en una curva que en realidad era elástica. Bajo los conceptos actuales de la cirugía, es de suma importancia determinar si una curva es estructural o no, ya que la recomendación es incluir en la artrodesis a la curva mayor junto a las estructurales, dejando libre aquellas curvas compensatorias; es decir hacer artrodesis selectivas.

Algunos cirujanos solicitan además radiografías con tracción axial de la columna, cuyo objetivo es determinar el grado de corrección de la deformidad mediante esta maniobra; son parte del estudio habitual en escoliosis neuromusculares y muchos cirujanos las utilizan en forma complementaria a las "side-bending"(13). Estas proyecciones se realizan también en posición supina y requieren de dos operadores: uno que traccione desde la cabeza y otro que traccione desde la pelvis o las extremidades inferiores.

\section{Evaluación de la radiografía}

El rol fundamental de las radiografías de columna total es la identificación y cuantificación de deformidades de la CV en los planos coronal y sagital.
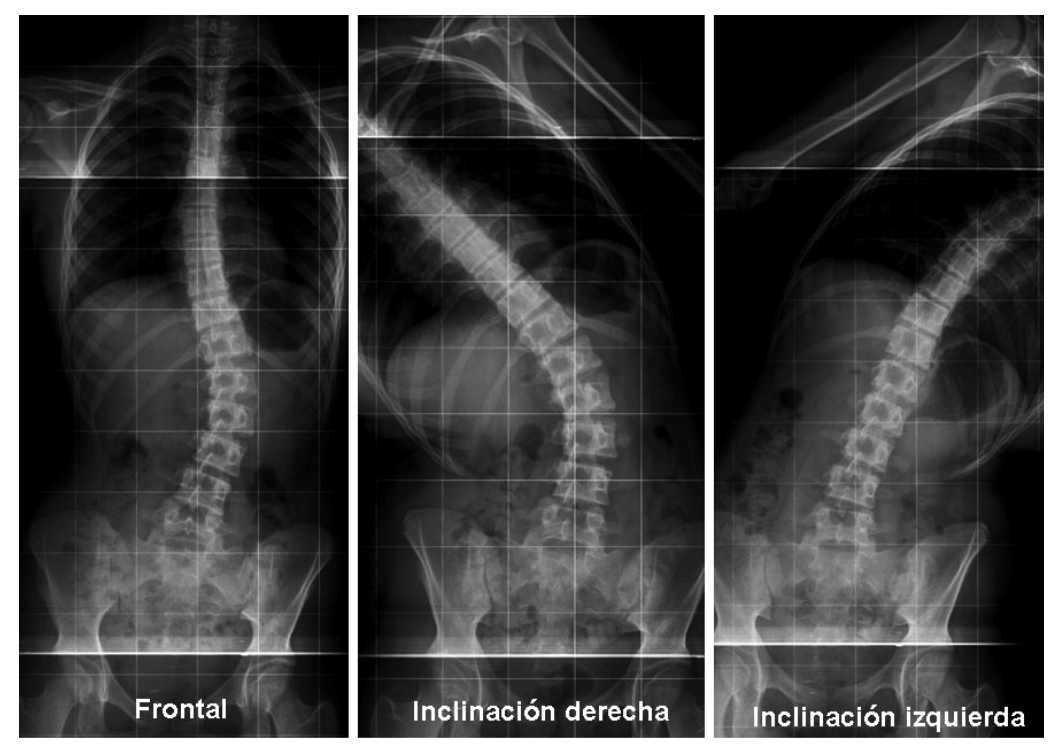

Figura 3. Telerradiografías de columna en proyecciones frontal neutra, con inclinación lateral o bending hacia derecha e izquierda, en que existe significativa corrección de las curvas torácica principal derecha y tóracolumbar izquierda.
Eventualmente se pueden detectar y caracterizar a grandes rasgos lesiones vertebrales (malformaciones, fracturas, lesiones tumorales, etc) así como cambios degenerativos.

Los dos factores principales que permiten predecir el riesgo de progresión de la escoliosis son: la severidad de la curva y el potencial de crecimiento esquelético, ambos factores evaluables a través de la radiología simple.

Para cuantificar correctamente una curva de escoliosis, deberíamos identificar:

- Las vértebras de ambos extremos de la curva, denominadas como vértebras proximal y distal, o terminales. Se eligen de manera que entre ellas formen el mayor ángulo posible y corresponden a aquellas que tienen la mayor inclinación hacia el lado cóncavo de la escoliosis, con la superficie del platillo vertebral mirando en este sentido. Cabe destacar que la curva se denominará según el lado al que apunte su convexidad(4,13) (Figura 4).

- El ápex de la curva escoliótica, que corresponde al punto con mayor desplazamiento lateral desde eje central de la columna (por ejemplo, línea plomada C7 o línea vertical centro-sacra, explicadas más adelante). Generalmente corresponde a una vértebra, la vértebra ápex o apical, que será la más rotada y desplazada lateralmente, pero también podría corresponder a un disco intervertebral ${ }^{(4,13)}$, como se observa en la Figura 4. 
El método de Cobb es el más difundido para la medición de las curvaturas fisiológicas o patológicas de la columna, tanto en los planos coronal y sagital, así como en las proyecciones con inclinación lateral(16). Inicialmente fue descrito para la medición de la escoliosis, midiendo el ángulo formado por la intersección de dos líneas trazadas paralelas al platillo superior de la vértebra proximal y al platillo inferior de la vértebra distal de la curva escoliótica; este es el método "directo" o "de dos líneas" (Figuras 4 y 5), útil y de elección en ángulos grandes. El método "indirecto" o "de cuatro líneas" (Figuras 4 y 5), mide el ángulo de la curva en la intersección de líneas perpendiculares trazadas a las líneas recién mencionadas (de los platillos superior e inferior de la curva escoliótica); esta técnica es útil en ángulos de menor magnitud, siendo necesario considerar que por la mayor utilización de líneas y ángulos se asocia a mayor variabilidad y error. Cabe destacar que, con los sistemas de radiología digital actual, todas las curvas son posibles de medir con el método de Cobb directo ${ }^{(10,12,20)}$.

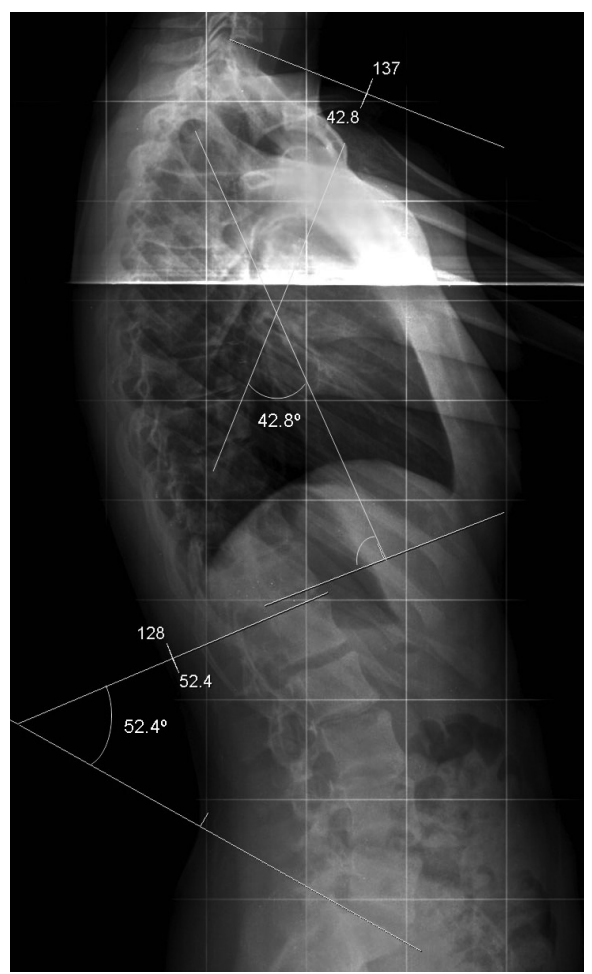

Figura 5. Radiografía lateral de columna con medición de la cifosis dorsal por el método de Cobb indirecto (con cuatro líneas) y la lordosis lumbar con el método de Cobb directo (dos líneas).

Para las curvas del plano sagital existe la posibilidad de medir el ángulo a los segmentos estrictos dorsal y lumbar (por ejemplo la cifosis dorsal entre el platillo vertebral superior de T1 e inferior de T12); sin embargo, se ha propuesto la utilización de niveles no-estrictos, que consideran los límites reales donde se extiende la curva cifótica o lordótica de la columna tóraco-lumbar. Este último concepto es el más congruente con la técnica original desarrollada por Cobb, que considera las vértebras más inclinadas como límite para la medición de la escoliosis en el plano coronal $^{(19)}$ (Figura 6). Es recomendable entonces, medir las curvas del plano sagital tanto en segmentos estrictos como no-estrictos. La cifosis torácica se medirá entre T4 y T12, la cifosis tóraco-lumbar entre T10 y L2 y la lordosis lumbar entre T12 y S1.

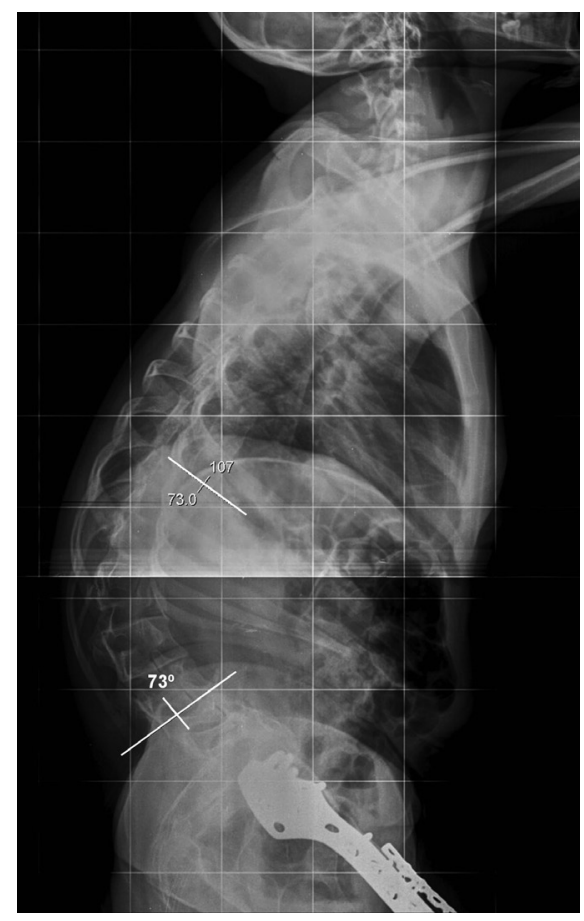

Figura 6. Radiografía lateral de columna total con medición de curva de cifosis tóraco-lumbar. Se ha utilizado un método de dos líneas y de tipo no estricto, tomando como límites las vértebras T11 y L4 en este caso particular.

Se considera que una curvatura mayor de $10^{\circ}$ en el plano coronal corresponde a escoliosis. Valores menores a $10^{\circ}$ se consideran "actitud" o "posición" escoliótica ${ }^{(4,13)}$. Según su magnitud, las curvas escolióticas pueden ser catalogadas en ${ }^{(10)}$ :

a) Leves: Curvas menores de $20^{\circ}$

b) Moderadas: Curvas de $20^{\circ}$ hasta $40^{\circ}$

c) Severas: Curvas mayores de $50^{\circ}$. La catalogación de las curvas entre $40^{\circ}$ y $50^{\circ}$ variará según el grado de madurez esquelética del paciente y también con la rigidez de las curvas evaluadas en las proyecciones con inclinación lateral (Figura 7).

La cifosis torácica es la curvatura posterior en el plano sagital a nivel torácico. Valores normales para este ángulo son variables en la literatura, debido principalmente a la limitación para la visualización de las vértebras torácicas altas en la radiografía lateral, 
por lo que los límites de las mediciones varían entre T1 y T5 por superior y T11 o T12 por inferior. El rango más frecuentemente aceptado es de $20-40^{\circ}(6,18)$ y la cifosis torácica máxima aceptada es de $50^{\circ}$.

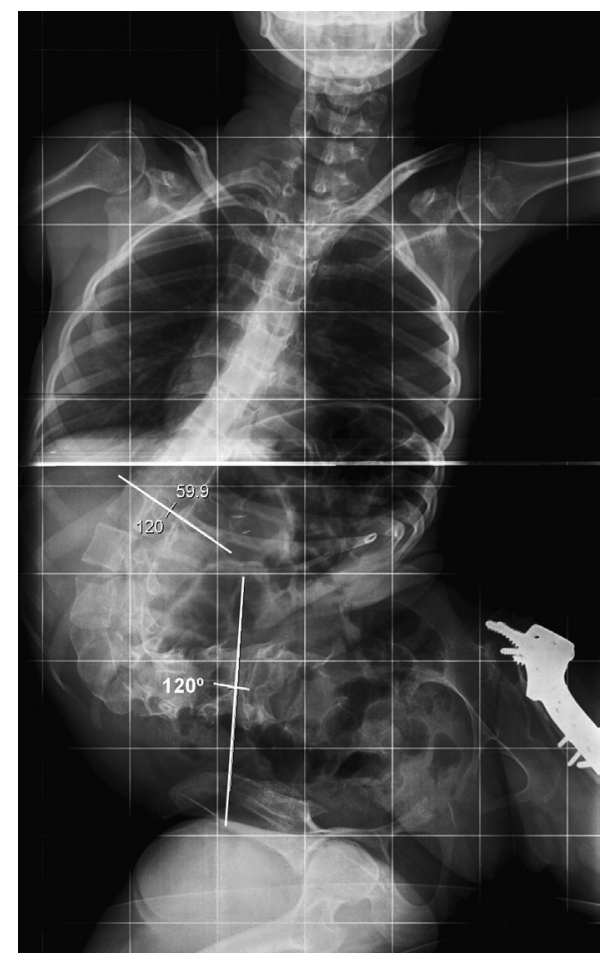

Figura 7. Radiografía AP de columna total mostrando una escoliosis severa tóracolumbar de convexidad derecha, su valor estimado con el método de Cobb de dos líneas es de $120^{\circ}$.

La lordosis lumbar es la convexidad anterior normal del segmento lumbar. El rango de medición también es variable en este caso; considerando como límite cefálico la plataforma inferior de T12 o la superior de L1, mientras que el límite caudal sería la plataforma inferior de L5 o la superior de S1. El rango de normalidad es muy amplio para este segmento: $-25^{\circ}$ a $-60^{\circ}$, siendo la mayor parte de la lordosis causada por los dos últimos niveles (L4 y L5). Los autores no han podido establecer diferencias claras en los valores entre personas sanas (sin síntomas) y pacientes con dolor y patología lumbar, aunque los pacientes con dolor lumbar tienden a tener una lordosis menor ${ }^{(5,20,21)}$.

Si bien la evaluación general de las deformidades de la CV se hace con el método de Cobb, también existen otros parámetros de utilidad que describiremos a continuación. La mayoría de éstos son aplicables tanto para niños como adultos, salvo el índice de Risser que se usa en pacientes en desarrollo puberal y, el ángulo costo-vertebral de Metha utilizado en escoliosis idiopáticas infantiles.

1. La línea vertical o plomada C7 (LPC7) se traza en la radiografía frontal o lateral desde el centro del cuerpo vertebral C7 hacia caudal, paralela al borde lateral de la radiografía (Figuras 8 y 9). Su utilidad radica en permitir una mejor identificación del ápex de las curvas (radiografía $(P)$ y es punto de referencia para la evaluación del balance coronal (radiografía AP) y sagital (radiografía lateral) de la $\mathrm{CV}^{(3)}$.

2. La línea vertical centro-sacra (LVCS) atraviesa el punto medio del platillo superior de S1, en sentido vertical y paralela al borde lateral de la radiografía frontal (Figura 8 ). Su utilidad radica en permitir una mejor identificación del ápex de las curvas y es punto de referencia para la evaluación del balance coronal de la columna vertebral (radiografía AP) ${ }^{(3)}$.

Figura 8. Radiografía frontal de columna total en que se ha trazado la línea plomada C7 (LPC7), desde el centro del cuerpo vertebral C7 y la línea vertical centrosacra (LVCS), que atraviesa el punto medio del sacro, ambas paralelas al borde lateral de la radiografía. En este paciente las líneas coinciden en su ubicación.

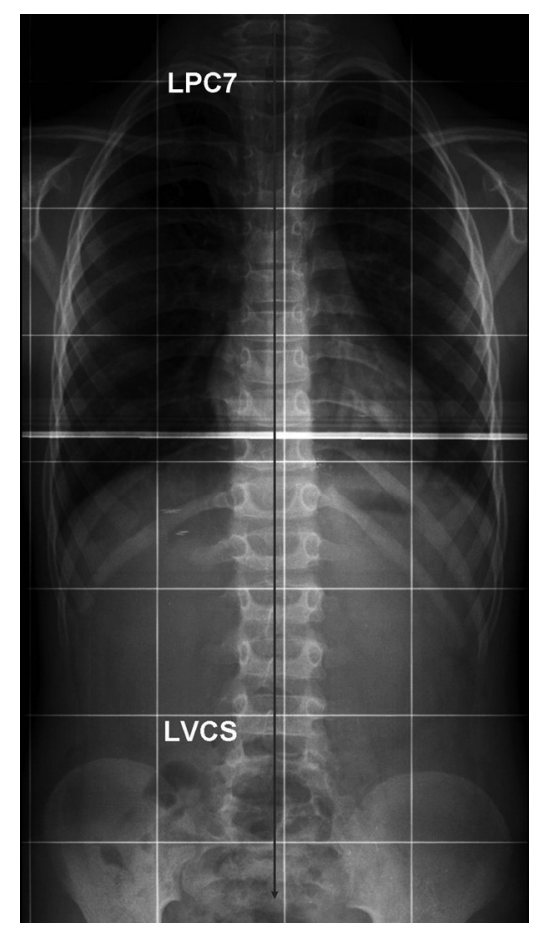

3. El balance espinal se refiere al equilibrio biomecánico de la columna vertebral, que en general debiera ser neutro, tanto en plano sagital como coronal. Este parámetro es especialmente relevante en pacientes candidatos a cirugía. Existen diversos métodos de evaluación del balance espinal sagital. Uno de ellos está determinado por la relación entre la LPC7 en la radiografía lateral y un punto de referencia, que corresponde a la esquina póstero-superior del cuerpo vertebral S1. Existe balance sagital positivo si la LPC7 pasa anterior a este punto de referencia y por el contrario, el balance es negativo si la vertical pasa por detrás de tal punto ${ }^{(3)}$ (Figuras 9 y 10$)$. 
4. El balance espinal coronal, evaluado en la proyección frontal, está determinado por la distancia horizontal entre la LPC7 y la LVCS. Si ambas líneas coinciden, el alineamiento coronal es neutro; cuando estas líneas no coinciden se habla de descompensación coronal, con balance positivo si la línea plomada queda a la derecha de la LVCS o, negativo si queda a la izquierda. Para algunos autores, el desbalance será significativo si existe una distancia de $\geq 3$ $\mathrm{cm}^{(3)}$ (Figura 11).

5. La traslación de la vértebra apical (TVA) de una curva escoliótica, es la distancia horizontal en el plano coronal entre el centro de la vértebra apical y el eje de la columna. En curvas torácicas altas y principales se mide hacia la LPC7 y en curvas tóraco-lumbares o lumbares la LVCS. La traslación es positiva si la vértebra apical está a la derecha y negativa si está a la izquierda ${ }^{(3)}$ (Figura 12). El valor obtenido refleja en forma indirecta el grado de deformidad del tronco y participa en la toma de decisiones de los niveles de artrodesis.

6. El ángulo costo-vertebral de Metha, de valor pronóstico para la progresión de las escoliosis infantiles, muestra la relación entre la vértebra apical de la curva escoliótica dorsal y la cabeza de sus costillas en la radiografía frontal. Se traza una línea perpendicular al borde inferior o superior de la vértebra apical de la curva escoliótica y una segunda línea desde el centro de la cabeza al punto medio del cuello de la costilla, medial a la región en que el cuello se ensancha; la intersección entre esta línea y la línea perpendicular de la vértebra forma el ángulo costo-vertebral. Una diferencia mayor de $20^{\circ}$ entre ambos lados es de mal pronóstico, con un riesgo de progresión de alrededor del $80 \%$ para estos casos, versus una progresión de alrededor del $20 \%$ en caso de una diferencia menor a $20^{\circ}(4)$ (Figura 13).
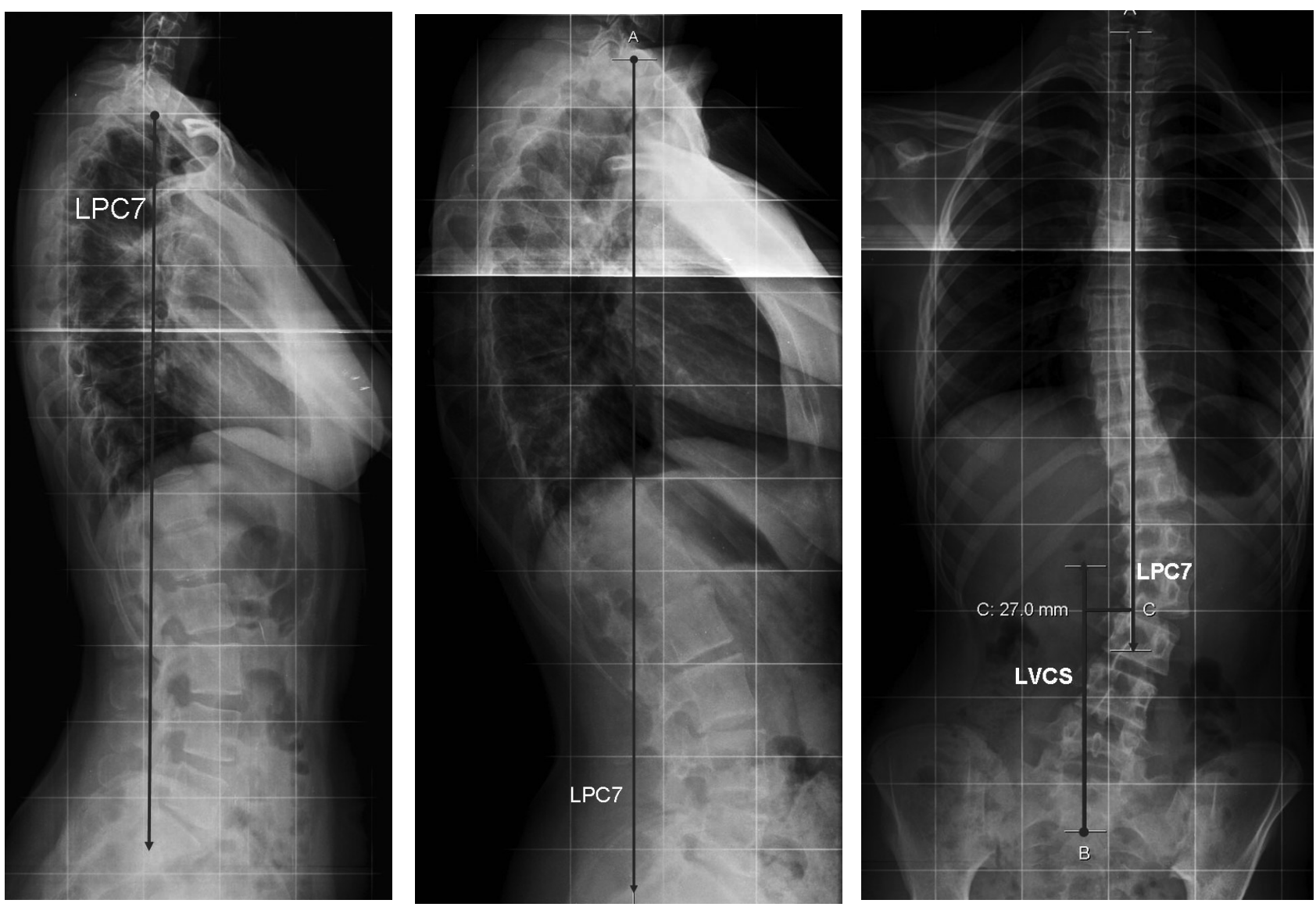

Figura 9. Proyección lateral que muestra la línea plomada C7 (LPC7) paralela al borde lateral de la radiografía, desde el centro del cuerpo vertebral C7 hacia caudal. El balance sagital es neutro si esta pasa por la esquina postero-superior de S1.
Figura 10. Radiografía lateral de columna total donde se ha marcado la línea LPC7, la cual pasa posterior a la esquina postero-superior del cuerpo vertebral S1, lo cual corresponde a un balance sagital negativo.
Figura 11. Paciente de sexo femenino de 18 años, con escoliosis con curva tóraco-lumbar izquierda y torácica principal derecha, en que existe falta de congruencia de las líneas LPC7 y LVCS, correspondiendo a un balance coronal negativo. 


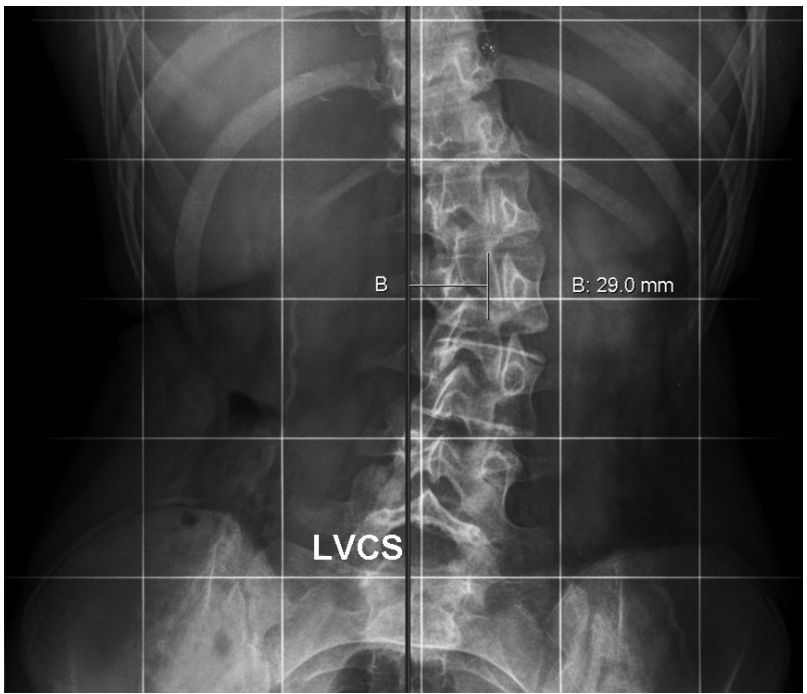

Figura 12. Medición de la traslación de vértebra ápex en curva escoliótica lumbar izquierda, en relación a la LVCS, clasificada como negativa por encontrarse a izquierda de la línea de referencia.

7. La evaluación de la rotación de los cuerpos vertebrales ha demostrado utilidad clínica en predecir la corrección, descompensación y progresión de la curva escoliótica. Habitualmente la vértebra apical es la que tiene el mayor grado de rotación. La medición por tomografía computada es el gold standard en exactitud. Sin embargo, su uso es limitado por costo, radiación y efecto del cambio en la curva escoliótica y rotación vertebral por la postura en decúbito.

En radiología simple destacaremos dos métodos de medición, cuya elección dependerá del grupo de trabajo ${ }^{(13,15)}$ :

- Método de Cobb, en el que se divide el ancho vertebral en sextos (tres a cada lado de la línea media) y se relaciona la apófisis espinosa con el centro del cuerpo vertebral. Se considera rotación positiva si la apófisis espinosa está rotada hacia la concavidad (lo habitual) y negativa si está desplazada hacia la convexidad. En el caso de rotación positiva, los valores serían,,$++++++y++++$ según el tercio que ocupe la apófisis espinosa; el grado ++++ esta por fuera del borde vertebral (Figura 14).

- El método de Nash y Moe relaciona el pedículo con el centro del cuerpo vertebral y clasifica de este modo la rotación vertebral en cuatro grados (Figura 15).

$\diamond$ Grado 0: ambos pedículos son simétricos.

$\checkmark$ Grado 1: pedículo situado hacia la concavidad de la escoliosis desplazado en dirección de este mismo margen del cuerpo vertebral.

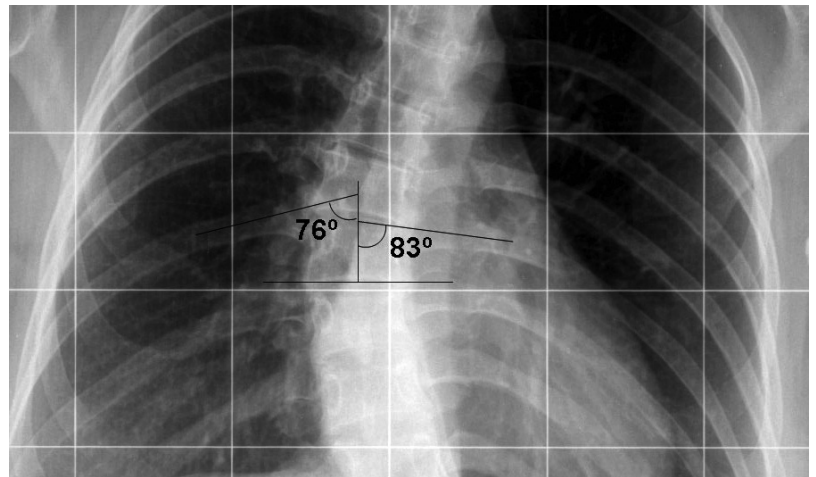

Figura 13. Medición del ángulo costo-vertebral de Metha en una escoliosis torácica principal derecha en la vértebra T8. El ángulo a derecha mide $76^{\circ}$ y a izquierda $83^{\circ}$, lo cual da una diferencia no significativa entre ambos lados.
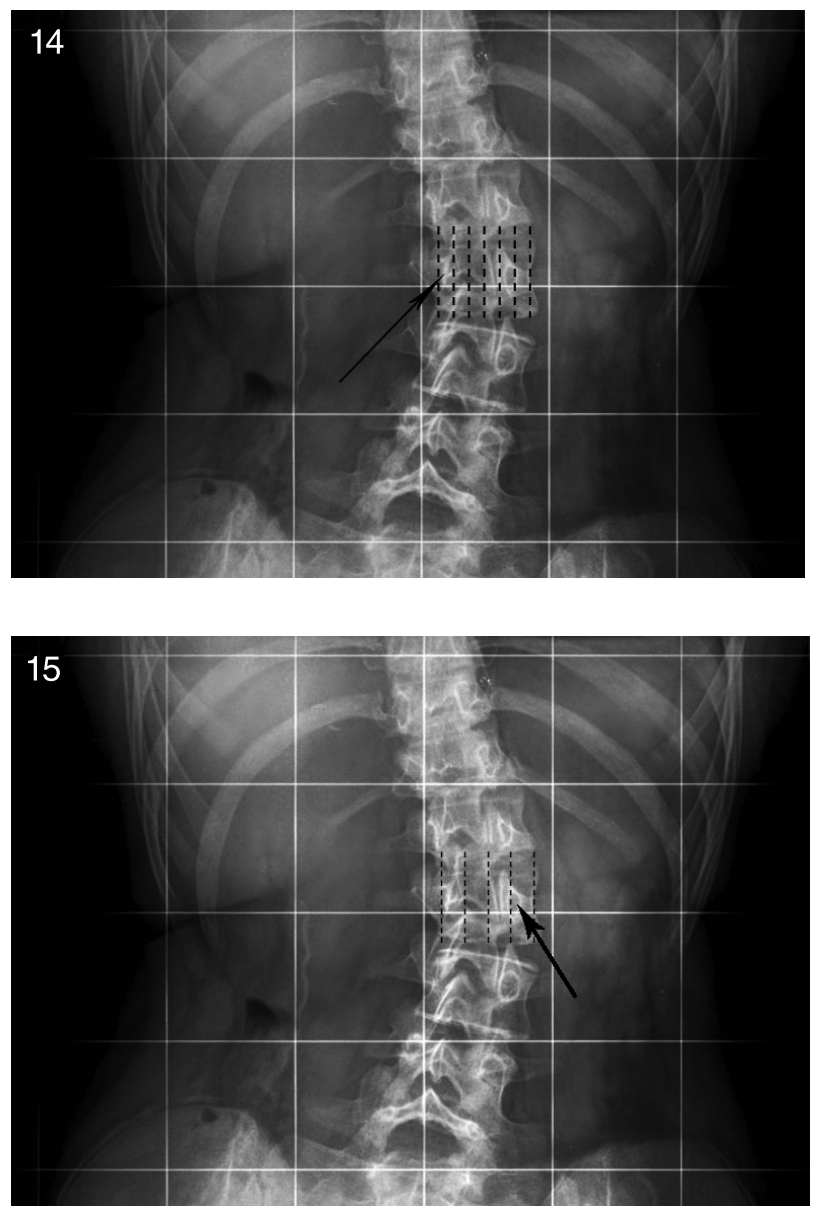

Figuras 14 y 15. Ejemplos de evaluación de la rotación vertebral en un mismo paciente, catalogado en +++ según el método de Cobb y de grado 2 según Nash-Moe.

$\diamond$ Grado 2: rotación entre 1 y 3, desaparición del pedículo del lado de la concavidad.

$\diamond$ Grado 3: pedículo del lado convexo en el centro del cuerpo vertebral.

$\diamond$ Grado 4: pedículo del lado convexo ha excedido la línea media. 
8. El índice de Risser para estimación de maduración ósea, evalúa el grado de aparición del núcleo de osificación de la cresta ilíaca y su fusión al ala ilíaca, como se observa en la Tabla I y en la Figura 16. El grado de madurez ósea es otro de los factores pronósticos de la escoliosis idiopática, existiendo una mayor progresión de la curva en niños con un grado de Risser 0 ó 1, que en grados 2 a 5 . La ventaja de utilizar este método es que las alas ilíacas están frecuentemente incluidas en la teleradiografía, por lo que sería innecesario contar con radiografías adicionales para la estimación de edad ósea ${ }^{(4)}$.

Tabla I. Indice de Risser ${ }^{(4,13)}$

Risser 0 Sin aparición de núcleo de osificación de la cresta ilíaca

Risser 1 Inicio de la osificación desde lateral, hasta $25 \%$

Risser 2 Id hasta 50\%

Risser 3 Id hasta $75 \%$

Risser $4 \quad 100 \%$ de osificación, aún sin fusión completa

Risser 5 Núcleo de osificación completamente fusionado

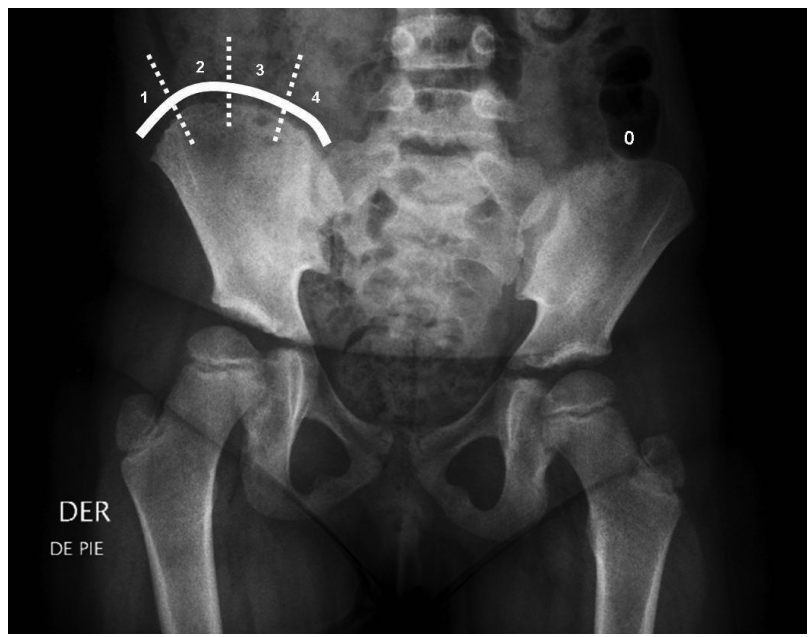

Figura 16. Indice de Risser. En un niño de 5 años aún no se identifica el núcleo de osificación de la cresta ilíaca (lado izquierdo), que equivale a un Risser 0 . A derecha se esquematiza la división en cuatro cuartos del ala ilíaca, correspondiendo el Risser 1 a la osificación dentro del primer cuarto y así sucesivamente. Risser 5 corresponde a la fusión del núcleo de osificación.

\section{Etiología, contexto clínico y clasificaciones de las escoliosis}

Las etiologías de las escoliosis son variadas; la diferencia principal se produce entre escoliosis idiopáticas y no idiopáticas. Así tenemos la siguiente clasificación etiológica ${ }^{(2):}$
- Idiopática (prácticamente el $80 \%$ de los casos)

- Congénita (alteraciones en la morfología vertebral por defectos de formación o segmentación, por ejemplo: vértebras en mariposa, hemivértebras, bloques vertebrales)

- Neuromuscular (por ejemplo: parálisis cerebral, poliomielitis, mielomenigocele, distrofias musculares).

- Deformidad vertebral secundaria a enfermedades sistémicas (por ejemplo: enfermedades reumatológicas)

- Deformidad vertebral secundaria a trauma o infección.

- Neurofibromatosis

- Tumoral

- Deformidades atribuibles a cambios relacionados con la edad (por ejemplo: patología degenerativa de $\mathrm{CV}$ ).

De acuerdo a la edad de inicio de las escoliosis idiopáticas, se clasifican en: inicio infantil (0- 3 años), inicio juvenil (3-10 años), escoliosis del adolescente (10 a 15 años y/o maduración ósea) y escoliosis del adulto (a partir de la maduración ósea) ${ }^{(4)}$. La incidencia de la escoliosis idiopática del adolescente (EIA), es del 2-3\% y es más frecuente en mujeres que en hombres. Escoliosis torácicas severas pueden reducir en forma significativa la capacidad cardiopulmonar y llegar a provocar un cor pulmonale. Esto no es así en las curvas tóracolumbares y lumbares, que pueden provocar dolor lumbar.

En escoliosis idiopáticas, el riesgo de progresión se basa fundamentalmente en dos factores: el grado de la curva y el grado de madurez esquelética; ambos datos se obtienen de la radiografía de columna total y son importantes de determinar. Se ha calculado la probabilidad de agravación en base al grado de la curva: en pacientes entre 10 y 12 años, las curvas bajo $20^{\circ}$ tienen una probabilidad de agravación de $25 \%$, curvas entre $20^{\circ}$ y $29^{\circ}$ tienen una probabilidad de agravación de $60 \%$, curvas entre $30^{\circ}$ y $60^{\circ}$ tienen $90 \%$ de probabilidad de agravarse $y$, aquellas curvas con más de $60^{\circ}$ se agravan en un $100 \%$. En pacientes esqueléticamente maduros, se agravan un $10 \%$ de las curvas entre $20^{\circ}$ y $29^{\circ}$, un $30 \%$ entre $30^{\circ}$ y $60^{\circ}$ y, $70 \%$ de aquellas curvas de más de $60^{\circ}$.

En el caso de las escoliosis no idiopáticas, la evolución es muy dependiente del tipo y etiología de la condición de base y la progresión tiende a ser más agresiva, con mayor compromiso funcional.

Con respecto a la clasificación de las escoliosis, se debe señalar que no existe un consenso, lo que se ha visto favorecido por el hecho que la escoliosis en los adolescentes es una patología de características diferentes a la escoliosis del adulto, especialmente por la presencia de componentes degenerativos en este último grupo. 
La clasificación de Lenke (2001) fue creada para la evaluación y planificación quirúrgica de las EIA ${ }^{(6,7)}$. Esta clasificación considera en primer lugar el patrón de curva, precisando los límites y nombres de las escoliosis según el segmento comprometido. De este modo, existen 4 segmentos importantes desde el punto de vista quirúrgico:

1. Torácico proximal: el ápex de la curva se encuentra entre el disco T2-T3 y el disco T5-T6.

2. Torácico principal: el ápex de la curva se encuentra entre la sexta vértebra torácica y el plano discal T11-T12.

3. Tóraco-lumbar: el ápex de la curva se encuentra entre el borde cefálico de la vértebra T12 y el borde caudal de L1.

4. Lumbar: el ápex de la curva se encuentra entre el plano discal L1-L2 y el borde caudal de la vértebra $L 4$.

Si bien en esta clasificación no se contemplan las escoliosis en niveles más altos, podemos delimitar otros dos niveles ${ }^{(4,13)}$ :

- Cervical: ápex de la curva de C1 a C6.

- Cervico-torácica: ápex de la curva de C7 a T1.

Para fines de la clasificación de Lenke la curva de mayor magnitud es siempre considerada estructural y se acompaña de curvas menores que pueden ser o no ser estructurales. Una curva menor es estructural si su magnitud es igual o mayor de $25^{\circ}$ en las proyecciones con inclinación lateral ${ }^{(3,6)}$. De este modo, Lenke describe 6 tipos de curvas ${ }^{(6)}$, como se describe en la Tabla II:

- Tipo 1: Torácica principal, la más frecuente, es la curva mayor. Las curvas torácica proximal y tóraco-lumbar/lumbar son menores y no estructurales (Figura 17).

- Tipo 2: Torácica doble, en la que la curva torácica principal es la curva mayor, mientras que la torácica proximal es menor y estructural. La curva tóraco-lumbar/lumbar es menor y no estructural.

- Tipo 3: Mayor doble, donde las curvas torácica
Figura 17. Ejemplo de un paciente con curva escoliótica torácica principal de convexidad derechay lumbar de convexidad i zquierda de menor severidad, tipo 1 de Lenke.

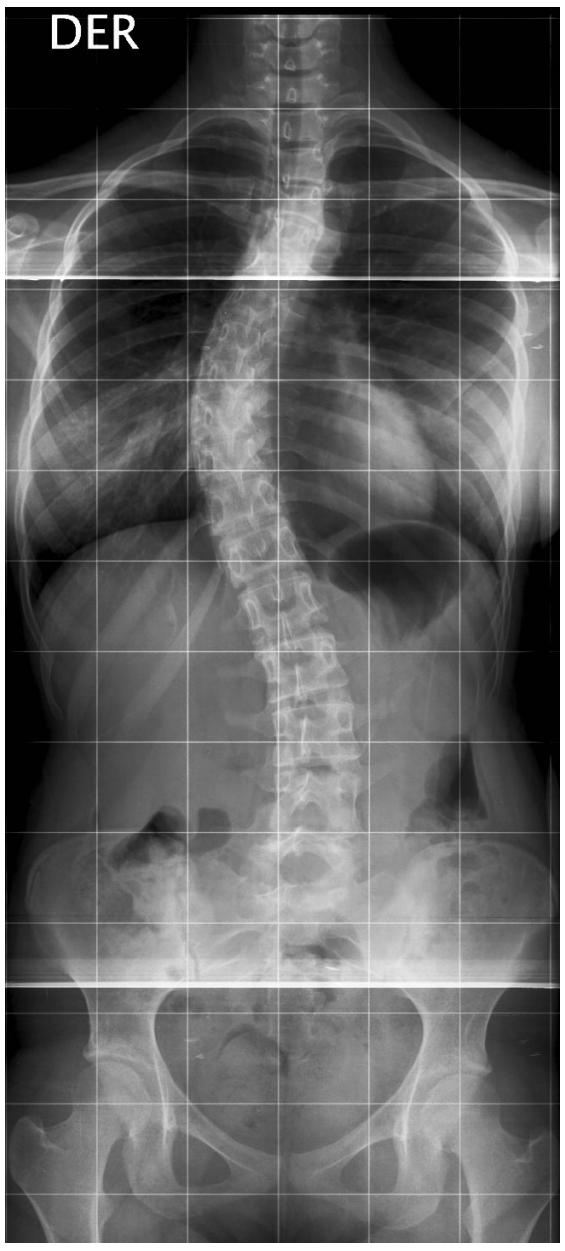

principal y tóraco-lumbar/lumbar son estructurales, mientras la curva torácica proximal es no estructural. La curva torácica principal es la mayor según el ángulo de Cobb o no más de $5^{\circ}$ menor que la curva tóraco-lumbar/ lumbar.

- Tipo 4: Mayor Triple, en la que las curvas torácica proximal, torácica principal y tóraco-lumbar/ lumbar son estructurales. Cualquiera de las dos últimas puede ser la curva mayor.

Tabla II. Clasificación de Lenke( ${ }^{(6)}$.

Patrones de curva

\begin{tabular}{|lccc|}
\hline Tipo de curva lumbar & Torácica proximal & Torácica principal & Tóraco-lumbar/lumbar \\
\hline Tipo 1 (torácica principal) & No estructural & Estructural & No estructural \\
Tipo 2 (torácica doble) & Estructural & Estructural & No estructural \\
Tipo 3 (mayor doble) & No estructural & Estructural & Estructural \\
Tipo 4 (mayor triple) & Estructural & Estructural & Estructural \\
Tipo 5 (tóraco-lumbar/lumbar) & No estructural & No estructural & Estructural \\
Tipo 6 (tóraco-lumbar/lumbar-torácica princ.) & No estructural & Estructural & Estructural \\
\hline
\end{tabular}

En cursiva y negrita se destaca la curva mayor en cada uno de los tipos de curva de esta clasificación. Nótese que, en el tipo 4 la curva mayor puede ser torácica principal o tóraco-lumbar/lumbar. 
- Tipo 5: Tóraco-lumbar/lumbar, donde la curva tóraco-lumbar/ lumbar es la mayor y es estructural. Las curvas torácica proximal y torácica principal son no estructurales.

- Tipo 6: Tóraco-lumbar/lumbar-torácica principal, en las que la curva tóraco-lumbar/ lumbar es la curva mayor y mide al menos $5^{\circ}$ más que la curva torácica principal, la que es estructural. La curva torácica proximal es no estructural.

La clasificación considera además modificadores, que no analizaremos en detalle en esta revisión, pero que son de importancia sólo si se está contemplando una resolución quirúrgica. Existen modificadores vertebrales lumbares que se basan en la relación de la LVCS con los cuerpos vertebrales lumbares y modificadores sagitales torácicos que se relacionan con el grado de cifosis. Respecto a este último punto, una curva de escoliosis menor será considerada estructural si la cifosis local es mayor de $20^{\circ}$ (por ejemplo, medida entre T2-T5 o T10-L2, para las curvas torácica alta y torácica principal/ tóraco-lumbar respectivamente).

La clasificación de las escoliosis en los adultos difiere un poco de la utilizada para los adolescentes. La Scoliosis Research Society-SRS (2007) propone la siguiente clasificación ${ }^{(7)}$ :

- Torácica simple

- Torácica doble

- Mayor doble

- Mayor triple

- Tóraco-lumbar

- Lumbar (idiopática o "de novo")

- Deformidad primaria en el plano sagital: aumento de la cifosis sin deformidad asociada en el plano coronal.

Las curvas torácicas son estructurales cuando miden $40^{\circ}$ o más, con una LPC7 pasando lateral al cuerpo vertebral del ápex de la curva. En el caso de curvas tóraco-lumbares y lumbares se consideran estructurales aquellas curvas que miden $30^{\circ}$ o más, con una LVCS pasando lateral al cuerpo vertebral del ápex de la curva.

Esta clasificación también incluye modificadores:

- Modificadores sagitales: aumento de las cifosis en columna tóraco-lumbar.

- Modificadores lumbares degenerativos: evidencias radiológicas de estrechamiento discal, espondiloartrosis y espondilolistesis degenerativa o subluxación rotatoria (Figura 18).

- Modificador de balance global: pérdida del balance sagital, con LPC7 ubicada $5 \mathrm{~cm}$ o más por delante o detrás del margen posterosuperior de S1. Pérdida del balance coronal, con LPC7 separada por $3 \mathrm{~cm}$ o más de la LVCS.

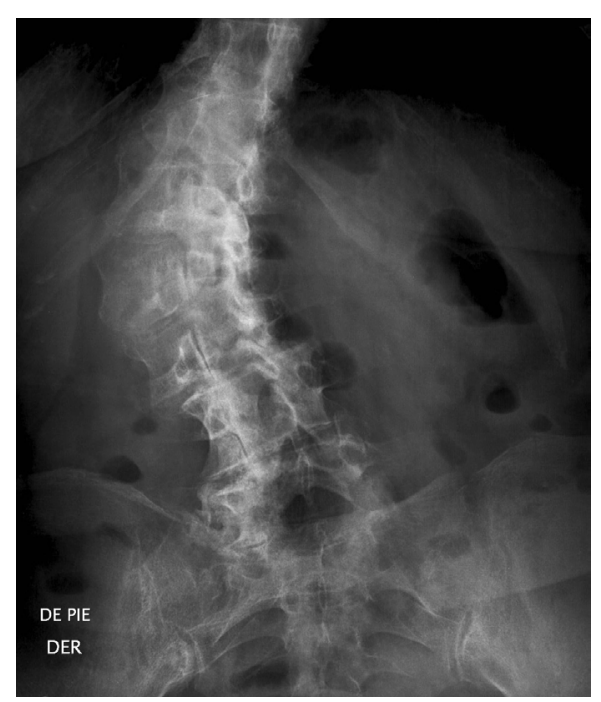

Figura 18. Radiografía AP en paciente femenino de 69 años, con escoliosis lumbar derecha, en la que existen también marcados cambios degenerativos caracterizados por espondiloartrosis y osteocondrosis.

\section{Aspectos generales en el manejo de la escoliosis}

El enfoque clínico difiere entre EIA y escoliosis desarrolladas en adultos; en el primer grupo, el objetivo del tratamiento es prevenir la progresión de la deformidad y limitar las consecuencias futuras de ésta, mientras que en los adultos es el manejo de la sintomatología actual y las incapacidades que ésta pueda generar. En el caso de adolescentes, un objetivo importante suele ser la corrección de la deformidad asociada para mitigar los efectos que ésta tiene sobre la imagen personal y autoestima, mientras que en el adulto el objetivo central es la recuperación funcional ${ }^{(7)}$.

A grandes rasgos, los tratamientos se dividen en ortopédicos y quirúrgicos. Dentro de los ortopédicos mencionaremos el corsé, que es un elemento que se utiliza en pacientes cuyo esqueleto es aún inmaduro, con curvas que cumplan el requisito de no ser muy severas (en general, bajo un Cobb de $40^{\circ}$ ) ni muy rígidas (muchos cirujanos considerarán como de baja posibilidad de éxito en la contención de la curva, aquellas en que una radiografía con corsé no logra disminuir la curva principal a aproximadamente un $50 \%$ del ángulo de Cobb sin corsé). Las cirugías tienen como objetivo fundamental evitar la progresión de la deformidad, lo que se consigue fusionando cuerpos vertebrales (artrodesis), para lo que existen diversos tipos de implantes metálicos. Otro objetivo importante de la cirugía es la corrección máxima de la deformidad, dentro de márgenes de seguridad ${ }^{(1,4,10)}$.

En el caso de los menores de 15 años con EIA y curvas menores de $25^{\circ}$, se recomienda observación de la progresión; para curvas entre $25^{\circ}$ y $40^{\circ}$ (con inmadurez esquelética), frecuentemente se opta por tratamiento con corsé. Las indicaciones quirúrgicas son 
variadas, pero en general en esqueletos inmaduros con curvas mayores de $40^{\circ}$ o maduros con más de $50^{\circ}$, la cirugía es una opción real de tratamiento. Cabe destacar que pacientes con escoliosis de $30^{\circ}$ y severas deformidades sagitales también pueden requerir cirugía. Para adultos jóvenes, 20-25 años, la indicación quirúrgica en general considera curvas de más de $50^{\circ}$ y/o que progresen en los controles ${ }^{(4,10,14)}$.

En los adultos, la causa degenerativa de la escoliosis es muy importante. En el cuadro clínico, es más trascendente el dolor y el compromiso neurológico que la deformidad propiamente tal. Los objetivos de la cirugía son la descompresión neural, la disminución del dolor y la corrección de la deformidad, con una enorme variabilidad en los manejos quirúrgicos propiamente tales. Según la sintomatología del paciente, escoliosis de magnitudes tan pequeñas como $15^{\circ}$ pueden llegar a ser intervenidas ${ }^{(8,9)}$.

\section{Conclusiones}

De la presente revisión hemos elaborado las siguientes conclusiones:

1. El principal objetivo de la radiografía de columna total es la caracterización de las deformidades de los ejes de la columna vertebral, su cuantificación y la estimación del grado de madurez esquelética, que son factores pronósticos trascendentes.

2. La cuantificación de las escoliosis es uno de los puntos centrales en el análisis de la radiografía de columna total. Los sistemas de radiología digital permiten realizar una medición sencilla y confiable, por lo que sus valores debieran ser consignados en los informes, respetando y utilizando la nomenclatura y los límites establecidos para la denominación de las curvas, según el segmento que afectan.

3. A pesar de la falta de clasificaciones de consenso para las escoliosis en adolescentes y adultos, existe gran similitud entre las dos principales clasificaciones, en los elementos radiológicos a evaluar, por lo que la generación de un lenguaje radiológico de consenso sería viable y conveniente de difundir.

4. La evaluación del balance sagital o coronal de la columna debiera incorporarse en el informe de estos exámenes.

\section{Bibliografía}

1. Grabowski G, Gelb D. Classification and treatment of adolescent idiopathic scoliosis. Curr Opin Orthop. 2005; 16: 158-162.

2. Thomsen M, Abel R. Imaging in scoliosis from the orthopaedic surgeon's point of view. Eur J Radiol. 2006; 58: 41-47.

3. Kuklo T. Radiographic evaluation of spinal deformity. Neurosurg Clin N Am. 2007; 18: 215-222.

4. González-Viejo M, Catalán M, Hijós M, Condón M.
Escoliosi idiopàtica: avaluació i tractament. Pediatr Catalana. 2000; 60: 142-149.

5. Fonseca L, Guarnieri S, Dorival A, Aparecido H. Lordose lombar: estudo dos valores angulares e da participação dos corpos vertebrais e discos intervertebrais. Acta Ortop Bras. 2006; 14(4): 143-148.

6. Lenke L, Betz R, Harms J, Bridwell K, Clements D, Lowe T and Blande K. Adolescent idiopathic scoliosis: A new classification to determine extent of spinal arthrodesis. J Bone Joint Surg Am. 2001; 83: 1169-1181.

7. Berven S, Lowe T. The scoliosis research society slassification for adult Spinal deformity. Neurosurg Clin N Am. 2007; 18: 207-213.

8. Faldini C, Pagkrati S, Grande G, Digennaro V, Faldini O, Giannini S. Degenerative lumbar scoliosis: features and surgical treatment. J Orthop Traumatol. 2006; 7: 67-71.

9. Berven S, Deviren V, Mitchell B, Wahba G, Hu S, Bradford D. Operative management of degenerative scoliosis: An evidence-based approach to surgical strategies based on clinical and radiographic outcomes. Neurosurg Clin N Am. 2007; 18: 261-272.

10. Protocolo AUGE. Tratamiento quirúrgico de la escoliosis en menores de 25 años. Gobierno de Chile, Ministerio de Salud. 2004: 1-20.

11. Vedantam R, Lenke L, Bridwell K, Linville D, Blanke K. The effect of variation in arm position on sagital spinal alignment. Spine. 2000; 25(17): 2204-2209.

12. Wills B, Auerbach J, Zhu X, Caird M, Horn D, Flynn J et al. Comparison of Cobb angle measurement of scoliosis radiographs with preselected end vertebrae. Traditional versus digital acquisition. Spine. 2007; 32 (1): 98-105.

13. Diard F, Chateil JF, Hauger O, Moinard M, DucouLePointe $\mathrm{H}$. Imagerie des scolioses de l'enfant et de l'adolescent. J Radiol. 2002; 83: 1117-1139.

14. Lenke L, Dobbs M. Management of juvenile idiopathic scoliosis. J Bone Joint Surg Am. 2007; 89A: 55-63.

15. Kuklo T, Potter B, Lenke L. Vertebral rotation and thoracic torsion in adolescent idiopathic scoliosis. What is the best radiographic correlate? J. Spinal Disord Tech. 2005; 182: 139-147.

16. Oda M, Rauh S, Gregory P, Silverman F, Bleck E. The significance of roentgenographic measurement in scoliosis. J Pediatr Orthop B. 1982; 24: 378-382.

17. Shea K, Stevens $P$, Nelson M, Smith J, Masters K, Yandow S. A comparison of manual versus computerassisted radiographic measurement: Intraobserver measurement variability for Cobb angles. Spine. 1998; 23(5): 551-555.

18. Macagno A, O'Brien M. Thoracic and thoracolumbar kyphosis in adults. Spine. 2006; 31(19): s161-170.

19. Mac-Thiong JM, Pinel-Giroux FM, de Guise J, Labelle H. Comparison between constrained and non-constrained Cobb techniques for the assessment of thoracic kyphosis and lumbar lordosis. Eur Spine J. 2007; 16 (9): 13251331.

20. Harrison D, Harrison D, Cailliet R, Jadik T, Holland B. Radiographic analysis of lumbar lordosis: Centroid, Cobb, TRALL and Harrison posterior tangent methods. Spine. 2001; 26(11): E235-242.

21. Cheng $X$, Sun $Y$, Boonen $S$, Nicholson $P$, Brys $P$, Dequeker $J$ et al. Measurements of vertebral shape by radiographic morphometry: Sex differences and relationships with vertebral level and lumbar lordosis. Skeletal Radiol. 1998; 27: 380-384. 